\title{
Geodesic acoustic modes excited by finite beta drift waves
}

Chakrabarti, Nikhil Kumar; Guzdar, P.N.; Kleva, R.G.; Naulin, Volker; Juul Rasmussen, Jens; Kaw, P.K

Published in:

Physics of Plasmas

Link to article, DOI:

$10.1063 / 1.3028311$

Publication date:

2008

Document Version

Publisher's PDF, also known as Version of record

Link back to DTU Orbit

Citation (APA):

Chakrabarti, N. K., Guzdar, P. N., Kleva, R. G., Naulin, V., Juul Rasmussen, J., \& Kaw, P. K. (2008). Geodesic acoustic modes excited by finite beta drift waves. Physics of Plasmas, 15(11), 112310.

https://doi.org/10.1063/1.3028311

\section{General rights}

Copyright and moral rights for the publications made accessible in the public portal are retained by the authors and/or other copyright owners and it is a condition of accessing publications that users recognise and abide by the legal requirements associated with these rights.

- Users may download and print one copy of any publication from the public portal for the purpose of private study or research.

- You may not further distribute the material or use it for any profit-making activity or commercial gain

- You may freely distribute the URL identifying the publication in the public portal 


\title{
Geodesic acoustic modes excited by finite beta drift waves
}

\author{
N. Chakrabarti, ${ }^{1}$ P. N. Guzdar, ${ }^{2}$ R. G. Kleva, ${ }^{2}$ V. Naulin, ${ }^{3}$ J. J. Rasmussen, ${ }^{3}$ \\ and P. K. Kaw ${ }^{4}$ \\ ${ }^{1}$ Saha Institute of Nuclear Physics, 1/AF Bidhannagar, Kolkata 700064, India \\ ${ }^{2}$ Institute for Research in Electronics and Applied Physics, University of Maryland, College Park, \\ Maryland 20742, USA \\ ${ }^{3}$ Association EURATOM-Ris $\phi$ DTU, Ris $\phi$ National Laboratory for Sustainable Energy, \\ Technical University of Denmark, P.O. 49, DK-4000 Roskilde, Denmark \\ ${ }^{4}$ Institute for Plasma Research, Bhat, Gandhinagar 384424, India
}

(Received 19 August 2008; accepted 29 October 2008; published online 25 November 2008)

Presented in this paper is a mode-coupling analysis for the nonlinear excitation of the geodesic acoustic modes (GAMs) in tokamak plasmas by finite beta drift waves. The finite beta effects give rise to a strong stabilizing influence on the parametric excitation process. The dominant finite beta effect is the combination of the Maxwell stress, which has a tendency to cancel the primary drive from the Reynolds stress, and the finite beta modification of the drift waves. The zonal magnetic field is also excited at the GAM frequency. However, it does not contribute to the overall stability of the three-wave process for parameters of relevance to the edge region of tokamaks. (C) 2008 American Institute of Physics. [DOI: 10.1063/1.3028311]

\section{INTRODUCTION}

Geodesic acoustic modes (GAMs) are a class of lowfrequency toroidal modes which are primarily electrostatic modes that are observed in a variety of tokamaks. ${ }^{1-7}$ These modes were first predicted theoretically by Winsor et $a .^{8}$ Their observation has stimulated theoretical and computational investigations to understand the excitation mechanism. In addition, the significance of the role of GAMs, observed in the edge region of tokamaks, in triggering the low to high confinement $(\mathrm{L}-\mathrm{H})$ transitions is an interesting question that needs to be addressed both observationally and theoretically. Presently there are two methods of excitation of modes in the GAM range of frequencies that are being investigated. The first is a linear process involving energetic ions. The lowfrequency chirps observed on Mirnov coils have been found near the GAM frequency and are believed to be excited by the neutral beam ions. ${ }^{9,10}$ The second class of modes near the GAM frequency are observed in the edge region of tokamaks by a variety of diagnostic methods, and these are believed to be excited by nonlinear processes. ${ }^{11-13}$ In this study we will focus on the nonlinear type of excitation and extend our earlier work by including finite beta effects.

\section{BASIC FINITE $\beta$ EQUATIONS}

The basic equations used in this investigation are those for finite beta drift waves and shear Alfvén waves (with diamagnetic drift effects). ${ }^{14-16}$ Here we use fluid equations to investigate the excitation of the GAMs by the nonlinear terms. In the edge region the contribution to the Landau damping is weak since the safety factor $q>3$ and hence kinetic effects will not contribute to the dynamics:

$$
\frac{d n}{d t}+\frac{\rho_{s} c_{s}}{L_{n}} \frac{\partial \phi}{\partial y}-2 \frac{\rho_{s} c_{s}}{R} \hat{C}(\phi-n)-\nabla_{\|} J=0,
$$

$$
\begin{aligned}
& \rho_{s}^{2} \nabla_{\perp} \cdot \frac{d}{d t} \nabla_{\perp} \phi+2 \frac{\rho_{s} c_{s}}{R} \hat{C} n-\nabla_{\|} J=0, \\
& \frac{\partial \psi}{\partial t}+\frac{\rho_{s} c_{s}}{L_{n}} \frac{\partial \psi}{\partial y}-v_{A} \nabla_{\|}(\phi-n)=0 .
\end{aligned}
$$

In these equations, the density, scalar, and vector potentials are normalized as follows: $n=\tilde{n} / n_{0}, \phi=e \widetilde{\phi} / T_{e 0}$, and $\psi$ $=v_{A} \tilde{\psi} / \rho_{s} c_{s} B_{0}$. Here, $\nabla_{\|}=\nabla_{\| 0}+\left(\rho_{s} c_{s} / v_{A}\right) \hat{z} \times \nabla \psi \cdot \nabla$ with $\nabla_{\| 0}$ $\sim(1 / q R)(\partial / \partial \theta)$ representing the parallel wave number in the equilibrium magnetic field. $J=\rho_{S}^{2} v_{A} \nabla_{\perp}^{2} \psi, \quad c_{s}^{2}=T_{e 0} / m_{i}, v_{A}^{2}$ $=B^{2} / 4 \pi m_{i} n=\Omega_{i}=e B_{0} / m_{i} c, \quad$ and $\quad \hat{C}=(\cos \theta / a)(\partial / \partial \theta)$ $+\sin \theta \partial / \partial x$. In addition, $d / d t=\partial / \partial t+\rho_{s} c_{s} \hat{z} \times \nabla \phi \cdot \nabla$. Here, $e$ is the electronic charge, $T_{e 0}$ is the equilibrium electron temperature, $a$ the minor radius, $m_{i}$ the mass of the ions, $L_{n}$ the density gradient scale-length, $c_{s}$ the ion acoustic speed, $v_{A}$ the Alfvén speed, and $\Omega_{i}$ the ion cyclotron frequency. $\hat{C}$ is the curvature operator, which includes both the normal curvature contribution (first term in $\hat{C}$ ) and the geodesic curvature. The parallel dynamics for the ions is neglected in this study since for GAMs it makes a minor contribution to the frequency in the edge region (where the safety factor $q$ is large).

To investigate the linear parametric excitation of GAMs by a "pump" drift wave the basic modes needed for this analysis is the pump wave, which for the present study is assumed to be a drift wave:

$$
\xi_{0}=\sum_{m} \xi_{m}^{0} e^{-i \omega_{0} t+i n \zeta+i m \theta} .
$$

Here, $\xi$ represents the density, scalar, and vector potential perturbations associated with the pump drift wave. The summation over $m$ is for a few symmetric sidebands around the primary mode number $m_{0}=n / q$, where $q$ is the safety factor 
at the rational surface. This mode can couple to the GAM mode, which is represented as

$$
\xi_{G}=\left[\phi_{G}, \psi_{G}, n_{G}(\theta)\right] e^{-i \omega t+i q_{r} x} .
$$

For the GAM, the potential is independent of the variable $\theta$, while the density has a $\sin \theta$ dependence. The GAM is primarily an electrostatic mode. So in a linear mode analysis there is no vector potential term which modifies the electrostatic dispersion relation. However, since we are exciting the GAM by a finite beta drift wave, the zonal magnetic field will be driven at the GAM frequency.

These two modes, namely, the pump drift wave and the GAM, excite a resonant sideband

$$
\xi_{s}=\sum_{m} \xi_{m}^{s} e^{-i\left(\omega-\omega_{0}\right) t-i n \zeta-i m \theta+i q_{r} x} .
$$

Using these representations, the equations for the driven GAM and sideband modes (retaining only the dominant nonlinear coupling) can be written as

$$
\begin{aligned}
& \left(\omega^{2}-\omega_{G}^{2}\right) \phi_{G}=-i \Gamma \omega_{G}\left(1-\frac{\omega_{0} \omega_{s}}{k_{\|}^{2} v_{A}^{2}}\right) \phi_{p} \phi_{s}, \\
& \omega \psi_{G}=i \Gamma \frac{\omega_{0} \omega_{s} \omega_{G}}{k_{\|}^{3} v_{A}^{3}} \phi_{p} \phi_{s}, \\
& {\left[\omega_{s}\left(1+k_{\perp}^{2} \rho_{s}^{2}\right)-\omega_{*}-\frac{\omega_{s}^{2}\left(\omega_{s}-\omega_{*}\right)}{k_{\|}^{2} v_{A}^{2}}\right] \phi_{s}} \\
& =i \Gamma F_{1} \phi_{p}^{*} \phi_{G}+i \Gamma F_{2} \phi_{p}^{*} \psi_{G},
\end{aligned}
$$

where

$$
\begin{aligned}
& \omega_{G}=\sqrt{2} c_{s} / R, \quad \Gamma=q_{r} k_{y} c_{s} \rho_{s}, \quad k_{\|}=1 / q R, \\
& \omega_{*}=k_{y} c_{s} \rho_{s} / L_{n}, \quad k_{y}=m_{0} / a, \quad \omega_{s}=\omega_{0}-\omega, \\
& k_{\perp}^{2}=q_{r}^{2}+\frac{m_{0}^{2}}{a^{2}},
\end{aligned}
$$

and

$$
\begin{aligned}
F_{1}= & {\left[1+\left(k_{y}^{2}-q_{r}^{2}\right) \rho_{s}^{2}\right]-\frac{k_{y}^{2}-q_{r}^{2}}{k_{\perp}^{2}}\left[\frac{\omega_{s}\left(\omega_{s}-\omega_{*}\right)}{k_{\|}^{2} v_{A}^{2}}\right] } \\
& -\frac{\omega_{0}\left(\omega_{0}+\omega_{s}-\omega_{*}\right)}{k_{\|}^{2} v_{A}^{2}}, \\
F_{2}= & -\frac{\omega_{s} \omega_{0}}{k_{\|}^{2} v_{A}^{2}}\left[\frac{\left(\omega_{0}-\omega_{*}\right)}{k_{\|} v_{A}}+\frac{\left(k_{y}^{2}-q_{r}^{2}\right)}{k_{\perp}^{2}} \frac{\left(\omega_{s}-\omega_{*}\right)}{k_{\|} v_{A}}\right] .
\end{aligned}
$$

As shown in earlier work, ${ }^{12}$ the dominant nonlinear coupling is to the primary mode $m_{0}=n / q$, where $q$ is the safety factor at the rational surface. There are couplings to the $m_{0} \pm 1$ sidebands, which turn out to be $1 / m_{0}$ smaller than the dominant terms. Equation (7) represents the excitation of the GAM by the beating of the pump wave $\phi_{p}$ and the sideband $\phi_{s}$. The second term on the right hand side arises from the finite $\beta$ effects of the drift wave branch. The zonal field $\psi_{G}$ [Eq. (8)] is driven by the pump and the sideband. This vector potential is nonresonant in character and therefore will not play a significant role in the excitation/suppression of the GAM. Nevertheless, it can be driven by the coupling process, and hence may be observed by pick-up coils which record the perturbed magnetic fields.

Finally, the equation for the drift wave sideband driven by the GAM and the pump wave is given by Eq. (9). Once again, the new terms in this calculation are the finite $\beta$ effects in the coupling coefficients in $F_{1}$ and $F_{2}$, which arise from the electromagnetic nature character of the pump and the drift wave, and the extra term on the right hand side due to coupling to the zonal field. This system of equations then can be analyzed to understand the excitation of GAMs by finite beta drift waves.

Making the approximation that $\omega=\omega_{G}+\delta \omega$, with $\delta \omega$ $<\omega_{G}$, the final dispersion relation for the excitation of GAMs by finite beta drift waves is given by

$$
\delta \omega^{2}+\gamma_{0}^{2}=0
$$

with

$$
\begin{aligned}
& \gamma_{0}^{2}=\frac{\Gamma^{2}\left|\phi_{p}\right|^{2} F_{1}}{2 A}\left(1-\frac{\omega_{0} \omega_{s 0}}{k_{\|}^{2} v_{A}^{2}}\right), \\
& A=1+k_{\perp}^{2} \rho_{s}^{2}-\omega_{s 0} \frac{\left(3 \omega_{s 0}-2 \omega_{*}\right)}{k_{\|}^{2} v_{A}^{2}},
\end{aligned}
$$

and $\omega_{s 0}=\omega_{0}-\omega_{G}$. If the sideband is a drift wave, the resonance condition demands that

$$
q_{r} \rho_{s}=\left[-1-k_{y}^{2} \rho_{s}^{2}+\frac{\omega_{*}}{\omega_{s 0}}+\frac{\omega_{s 0}\left(\omega_{s 0}-\omega_{*}\right)}{k_{\|}^{2} v_{A}^{2}}\right]^{1 / 2} .
$$

This condition therefore selects a specific value of the radial wave-vector $q_{r}$ of the GAM which will be preferentially excited. Furthermore, the dispersion relation [Eq. (10)] reduces to the earlier electrostatic result ${ }^{12}$ in the limit $v_{A} \rightarrow \infty$. There are a number of finite beta modifications in this dispersion relation. The finite beta corrections in the terms $F_{1}, F_{2}$, and $A$ are due the finite beta nature of the pump and sideband drift waves. Another important finite beta effect is the term $C$, the second term inside the bracket. This is the contribution from the Maxwell stress, which tends to cancel the first term from the Reynolds stress. This competition between these two stresses has been observed and investigated in many simulations which examine the energetics of zonal flow generation by a combination of Reynolds stress, Maxwell stress, and geodesic curvature drives. ${ }^{17-19}$ In the next section we evaluate the dispersion relation for typical tokamak plasma edge parameters.

\section{NUMERICAL RESULTS}

As in earlier work on the excitation of zonal flows by finite beta drift waves ${ }^{20}$ to evaluate the growth rate for the excitation of the GAMs and the effect of the finite beta, following normalization is used. For the pump wave, the frequency is normalized to the drift frequency. Hence, the normalized dispersion relation for the pump is 


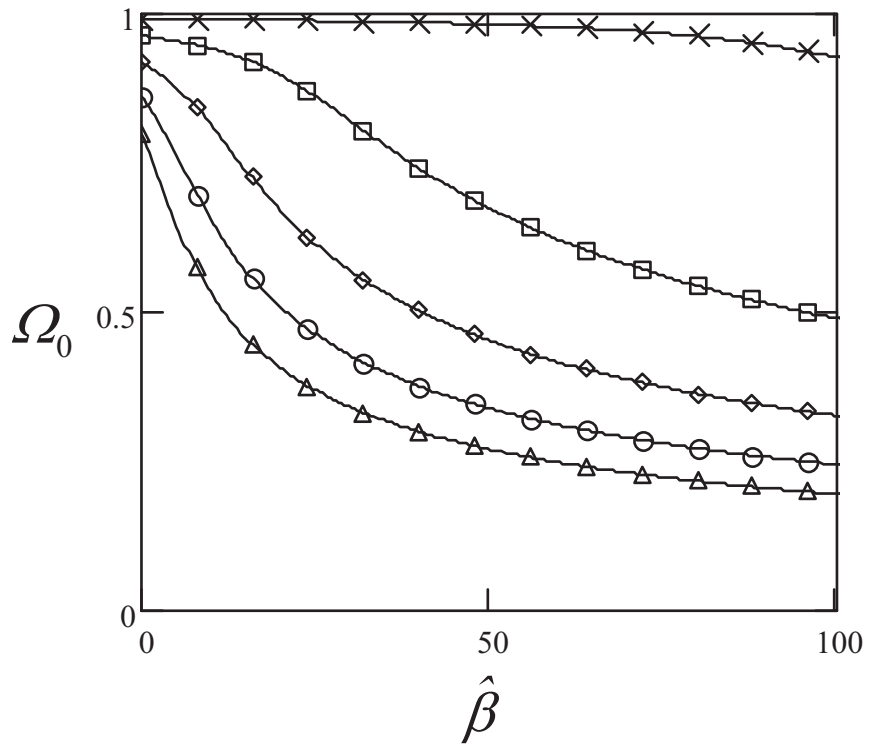

FIG. 1. Pump wave frequency as a function of $\hat{\beta}$ for $\hat{k}_{y}=0.1$ (crosses), 0.2 (squares), 0.3 (diamonds), 0.4 (circles), and 0.5 (triangles), respectively.

$$
1+\hat{k}_{y}^{2}-\frac{1}{\Omega_{0}}-\hat{k}_{y}^{2} \hat{\beta} \Omega_{0}\left(\Omega_{0}-1\right)=0 .
$$

There are basically two dimensionless parameters, (1) $\hat{k}_{y}$ $=k_{y} \rho_{s}$ and (2) $\hat{\beta}=\beta q^{2} R^{2} / 2 L_{n}^{2}$, where $\beta$ is the ratio of plasma pressure (electron) to the magnetic field pressure. In Fig. 1 is shown the drift wave branch as a function of $\hat{\beta}$ for $\hat{k}_{y}$ $=0.1,0.2,0.3,0.4,0.5$.

Now, from the resonance condition for the three-wave interaction, the radial wave number given by Eq. (11) can be evaluated. The normalized version of Eq. (11) with $\hat{q}_{r}$ $=q_{r} \rho_{s}$ is

$$
\hat{q}_{r}=\left[-1-\hat{k}_{y}^{2}+\frac{1}{\Omega_{s 0}}+\hat{k}_{y}^{2} \hat{\beta} \Omega_{s 0}\left(\Omega_{s 0}-1\right)\right]^{1 / 2} .
$$

Here, $\Omega_{s 0}=\Omega_{0}-\Omega_{G}$ with $\Omega_{G}=\sqrt{2} \varepsilon_{n} / \hat{k}_{y}$ the normalized GAM frequency and $\varepsilon_{n}=L_{n} / R$.

Finally, the normalized dispersion relation for excitation of GAMs can be written as

$$
\delta \Omega^{2}+\hat{\gamma}_{0}^{2}=0
$$

with

$$
\begin{aligned}
\hat{\gamma}_{0}^{2}= & \frac{\left(1-\Omega_{0} \Omega_{s 0} \bar{\beta}\right)}{2 \hat{A}}\left\{1-\Omega_{0}\left(\Omega_{s 0}+\Omega_{0}-1\right) \bar{\beta}\right. \\
& \left.-\frac{\left(\hat{k}_{y}^{2}-\hat{q}_{y}^{2}\right)}{\hat{k}_{\perp}^{2}}\left[\Omega_{s 0}\left(\Omega_{s 0}-1\right) \bar{\beta}\right]\right\}, \\
\hat{A}=1+\hat{k}_{\perp}^{2}-\Omega_{s 0}\left(3 \Omega_{s 0}-2\right) \bar{\beta}, &
\end{aligned}
$$
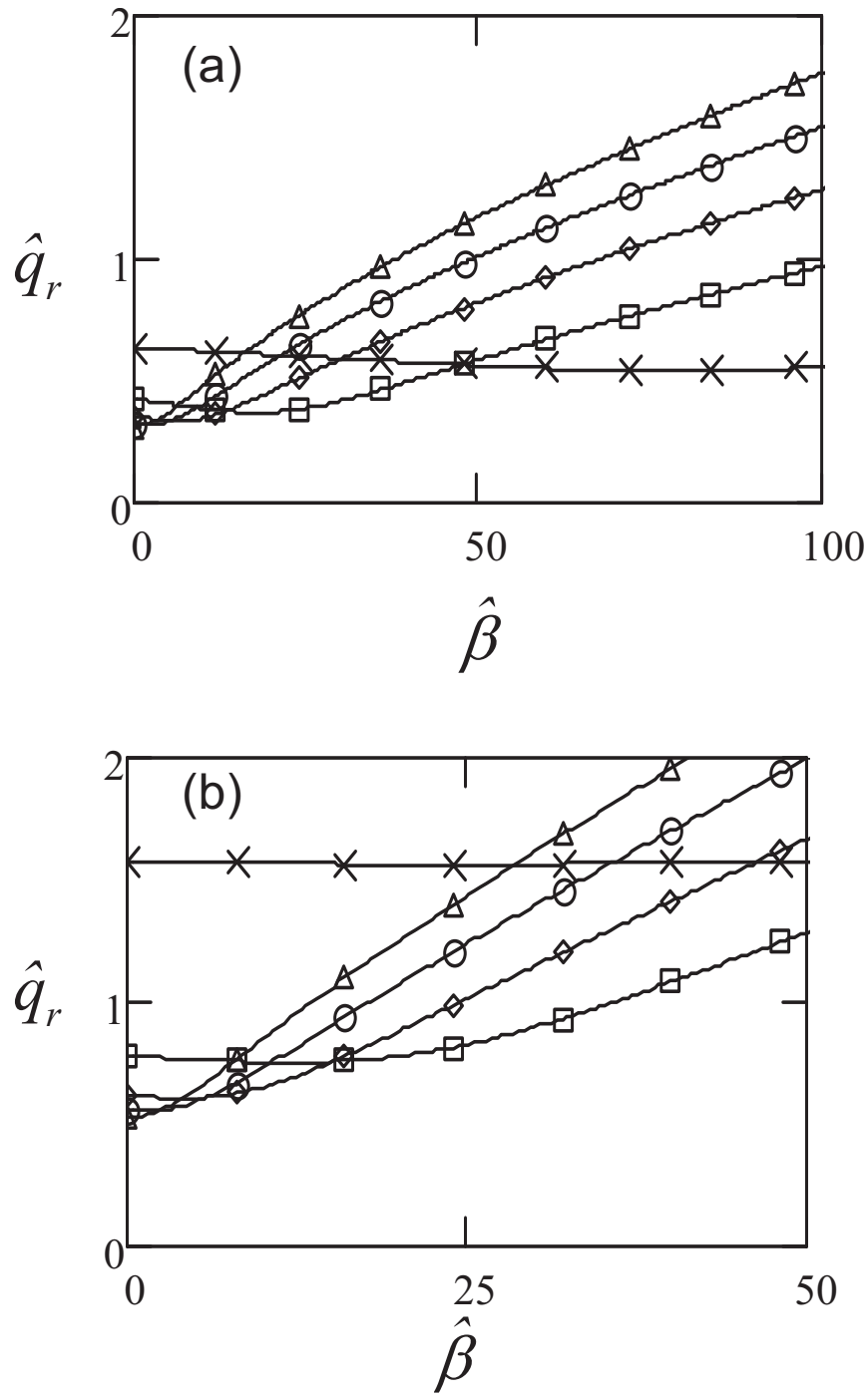

FIG. 2. Radial wave number $\hat{q}_{r}$ [Eq. (13)] as a function of $\hat{\beta}$ and $\hat{k}_{y}=0.1$ (crosses), 0.2 (squares), 0.3 (diamonds), 0.4 (circles), and 0.5 (triangles), respectively, for (a) $\varepsilon_{n}=0.02$ and (b) $\varepsilon_{n}=0.05$.

$$
\hat{\phi}_{p}=\frac{L_{n}}{\rho_{s}} \phi_{p}, \quad \delta \Omega=\frac{\delta \omega L_{n}}{\hat{q}_{r} \hat{k}_{y} c_{s}\left|\hat{\phi}_{p}\right|}, \quad \bar{\beta}=\hat{k}_{y}^{2} \hat{\beta} .
$$

In Fig. 2, $\hat{q}_{r}$ given by Eq. (13) is plotted as a function of $\hat{\beta}$ for the same five values of $\hat{k}_{y}$ as in Fig. 1 and for (a) $\varepsilon_{n}$ $=0.02$ and (b) $\varepsilon_{n}=0.05$. For larger $\varepsilon_{n}$, the radial wave number shifts to larger values. In fact, for corelike $\varepsilon_{n} \sim 1$, the resonance condition is not satisfied and hence the mechanism being investigated in this paper in the local limit does not excite modes in the core region of tokamaks.

Finally, the normalized growth rate $\delta \hat{\Omega}=\hat{q}_{r} \hat{k}_{y} \delta \Omega$ $=\delta \omega L_{n} / c_{s}\left|\hat{\phi}_{p}\right|$ with $\hat{\phi}_{p}=0.01$ for the excitation of the GAMs is plotted in Fig. 3 as a function of $\hat{\beta}$ for the same values $\hat{k}_{y}$ as in Fig. 1 for (a) $\varepsilon_{n}=0.02$ and (b) $\varepsilon_{n}=0.05$. There are various interesting features that can be gleamed from these plots. Each of the traces shows that with increasing $\hat{\beta}$ the growth rate for the GAM is suppressed and is completely stabilized beyond a critical value of $\hat{\beta}=\hat{\beta}_{C}$, which increases with $\hat{k}_{y}$. The dominant finite beta effect which is responsible 

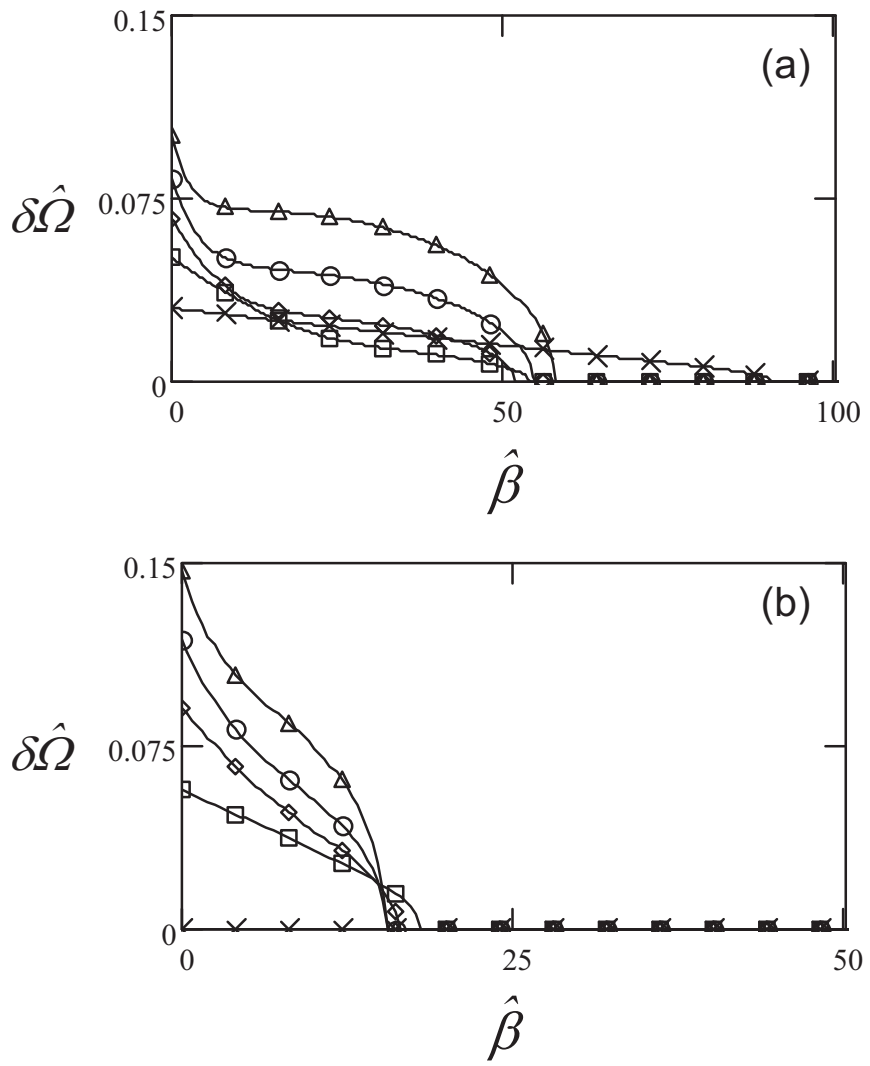

FIG. 3. Normalized growth rate $\delta \hat{\Omega}$ as a function of $\hat{\beta}$ for $\hat{\phi}_{p}=0.01$ and $\hat{k}_{y}=0.1$ (crosses), 0.2 (squares), 0.3 (diamonds), 0.4 (circles), and 0.5 (triangles), respectively, and (a) $\varepsilon_{n}=0.02$ and (b) $\varepsilon_{n}=0.05$.

for the stabilization is a combination of the Maxwell stress term and the finite beta modifications of the drift waves, which lead to the appearance of the $\hat{\beta}$-dependent terms in $F_{1}$. The zonal field gets excited by the mode coupling but plays no role in the growth (or suppression) of the GAM mode. Finally, plotted in Fig. 4 is the stability boundary $\hat{\beta}_{c}$ in the $\left(\hat{\beta}, \hat{k}_{y}\right)$ space $\left(\hat{\beta}_{c}\right.$ is the critical value of $\hat{\beta}$ above which the GAMs excitation is suppressed by finite beta effects) for four different values of $\varepsilon_{n}$.

For each value of $\varepsilon_{n}$, the GAMs can be excited by coupling to finite beta drift waves for values of $\hat{\beta}$ below the corresponding critical curve. Above the curve, the excitation by the resonant parametric process is suppressed by finite beta effects. Nevertheless, as can be seen from Fig. 4, GAMs can be excited over a broad range of parameters relevant to the edge region of tokamaks by the process studied in this paper. Qualitatively one can see that for reasonable values of the low (L) mode edge parameters, namely, $\varepsilon_{n}=0.05$, the GAMs are strongly stabilized for rather low values of $\hat{\beta}$. Hence, they may become stable before the discharge reaches values of $\hat{\beta}$ for a $\mathrm{L}-\mathrm{H}$ transition. There are interesting observations on Doublet III-D (DIII-D), ${ }^{21}$ which show GAM suppression ${ }^{22}$ before the discharge reaches the parameters at which the L-H transition occurs. However, a more careful investigation of this issue and comparison with data will be undertaken in the future.

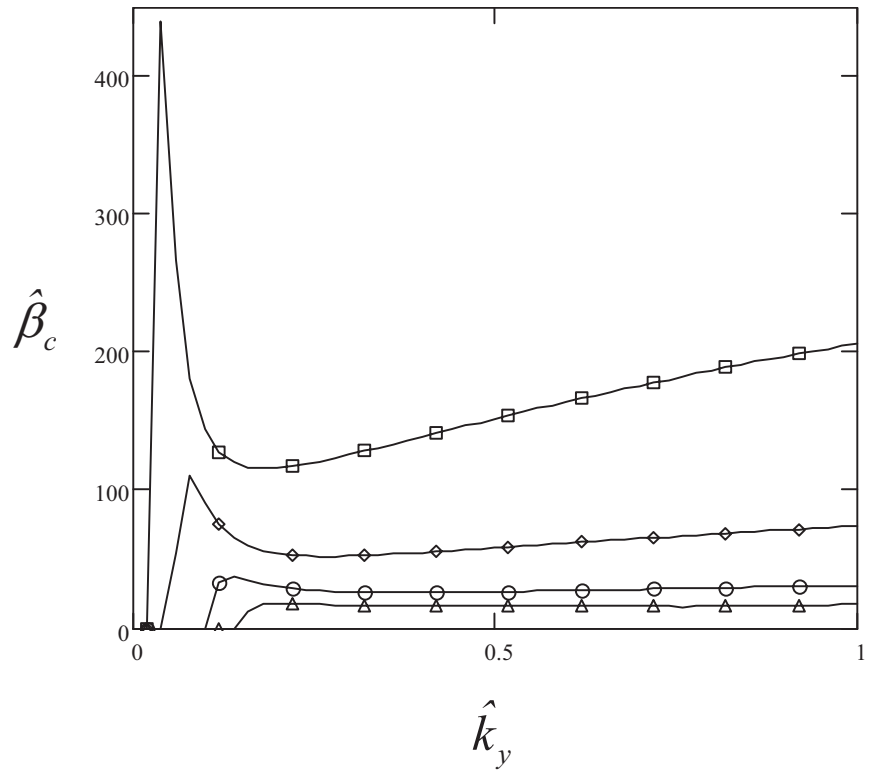

FIG. 4. $\hat{\beta}_{c}$ for $\hat{\phi}_{p}=0.01$ as a function of $\hat{k}_{y}$ for $\varepsilon_{n}=0.01$ (squares), 0.02 (diamonds), 0.035 (circles), and 0.05 (triangles).

\section{CONCLUSIONS}

In this paper, we have presented calculations which address the issue of excitation of the geodesic acoustic mode by nonlinear mode-coupling to finite $\beta$ drift waves. This is an extension of our earlier work in which the modes were primarily electrostatic. The present study shows that the inclusion of the finite beta effects lead to stabilization of the three-wave resonant process as the dimensionless parameter $\hat{\beta}$ increases. There are three dimensionless parameters in the present problem; namely, $\hat{\beta}, \varepsilon_{n}$, and $\hat{k}_{y}$. The resonance condition determines the radial wave number of the excited GAM as a function of these three dimensionless parameters. The dominant stabilization arises from a combination of the Maxwell stress associated with the finite beta modification of the drift waves (both pump and sideband). Also due to the finite beta effect there is the excitation of a zonal field at the GAM frequency. Though this field does not contribute to the overall stability of the GAM excitation, it can provide a magnetic signature for the GAM which is excited by the proposed mechanism. Finally, the stability boundary in the three-dimensional space $\hat{\beta}, \varepsilon_{n}, \hat{k}_{y}$ is computed.

\section{ACKNOWLEDGMENTS}

The work of P.N.G. was supported by a grant from the U.S. Department of Energy. V.N. and J.J.R. were supported by the Danish Natural Science Research Council (Contract No. FNU-272-06-0367).

${ }^{1}$ G. R. McKee, R. J. Fonck, M. Jakubowski, K. H. Burrell, K. Hallatschek, R. A. Moyer, D. L. Rudakov, W. Nevins, G. D. Porter, P. Schoch, and X. Xu, Phys. Plasmas 10, 1712 (2003).

${ }^{2}$ H. Punzmann and M. G. Shats, Phys. Rev. Lett. 93, 125003 (2004).

${ }^{3}$ G. Conway, B. Scott, J. Schirmer, M. Reich, A. Kendl, and the ASDEX Upgrade Team, Plasma Phys. Controlled Fusion 47, 1165 (2005).

${ }^{4}$ T. Ido, Y. Muira, K. Kamiya, Y. Hamada, K. Hoshino, A. Fujisawa, K. Itoh, S.-I. Itoh, A. Nishizawa, H. Ogawa, Y. Kusama, and JFT-2M group, 
Plasma Phys. Controlled Fusion 48, S41 (2006).

${ }^{5}$ G. R. McKee, D. K. Gupta, R. J. Fonck, D. J. Schlossberg, M. W. Shafer, and P. Gohil, Plasma Phys. Controlled Fusion 48, S123 (2006).

${ }^{6}$ A. V. Melnikov, V. A. Vershkov, L. G. Eliseev, S. A. Grashin, A. V. Gudoznik, L. I. Krupnik, S. E. Lysenko, V. A. Mavrin, S. V. Perfilov, D. A. Shelukin, S. V. Soldatov, M. V. Ufimtsev, A. O. Urazbaev, G. Van Oost, and L. G. Zimeleva, Plasma Phys. Controlled Fusion 48, S87 (2006).

${ }^{7}$ K. J. Zhao, T. Lan, J. Q. Dong, L. W. Lan, W. Y. Hong, C. X. Yu, A. D. Liu, J. Qian, J. Cheng, D. L. Yu, Q. W. Yang, X. T. Ding, Y. Liu, and C. H. Pan, Phys. Rev. Lett. 96, 255004 (2006).

${ }^{8}$ N. Winsor, J. L. Johnson, and J. M. Dawson, Phys. Fluids 11, 2448 (1968).

${ }^{9}$ C. J. Boswell, H. L. Berk, D. N. Borba, T. Johnson, S. D. Pinches, and S. E. Sharapov, Phys. Lett. A 358, 154 (2006).

${ }^{10}$ H. L. Berk, C. J. Boswell, D. Borba, A. C. A. Figueiredo, T. Johnson, M. F. F. Nave, S. D. Pinches, S. E. Sharapov, and the JET EFDA contributors, Nucl. Fusion 46, S888 (2006)

${ }^{11}$ K. Itoh, K. Hallatschek, and S. Itoh, Plasma Phys. Controlled Fusion 47, 451 (2005)

${ }^{12}$ N. Chakrabarti, R. Singh, P. K. Kaw, and P. N. Guzdar, Phys. Plasmas 14, 052308 (2007)

${ }^{13}$ F. Zonca and L. Chen, Europhys. Lett. 83, 35001 (2008).

${ }^{14}$ A. Zeiler, J. F. Drake, and B. Rogers, Phys. Plasmas 4, 2134 (1997).

${ }^{15}$ B. D. Scott, Plasma Phys. Controlled Fusion 39, 1635 (1997).

${ }^{16}$ V. Naulin, Phys. Plasmas 10, 4016 (2003).
${ }^{17}$ B. D. Scott, New J. Phys. 7, 92 (2005).

${ }^{18}$ V. Naulin, A. Kendl, O. E. Garcia, A. H. Nielsen, and J. J. Rasmussen, Phys. Plasmas 12, 052515 (2005).

${ }^{19}$ R. G. Kleva and P. N. Guzdar, Phys. Plasmas 15, 082307 (2008).

${ }^{20}$ P. N. Guzdar, R. G. Kleva, A. Das, and P. K. Kaw, Phys. Rev. Lett. 87, 015001 (2001)

${ }^{21}$ J. L. Luxon, R. Anderson, F. Batty, C. B. Baxi, G. Bramson, N. H. Brooks, B. Brown, B. Burley, K. H. Burrell, R. Callis, G. Campbell, T. N. Carlstrom, A. P. Colleraine, J. Cummings, L. Davis, J. C. DeBoo, S. Ejima, R. Evanko, H. Fukumoto, R. Gallix, J. Gilleland, T. Glad, P. Gohil, A. Gootgeld, R. J. Groebner, S. Hanai, J. Haskovec, E. Heckman, M. Heilberger, F. J. Helton, N. Hosogane, C.-L. Hseih, G. L. Jackson, G. Jahns, G. Janeschitz, E. Johnson, A. G. Kallman, J. S. Kim, J. Kohli, A. Langhorn, L. L. Lao, P. Leek, S. Lightner, J. Lohr, M. A. Mahdavi, M. Mayberry, B. McHarg, T. McKelvey, R. Miller, C. P. Moeller, D. Moore, A. Nerem, P. Noll, T. Okhawa, N. Ohyabu, T. H. Osborne, D. O. Overskei, P. I. Petersen, T. W. Petrie, J. Phillips, R. Prater, J. Rawls, E. E. Reis, D. Remsen, P. Riedy, P. Rock, K. Schaubel, D. P. Schissel, J. T. Schoville, R. Seraydarian, M. Shimada, T. Shoji, B. Sleaford, J. P. Smith, Jr., T. Smith, R. T. Snider, R. D. Stambaugh, R. Stav, H. St. John, R. E. Stockdale, E. L. Strait, R. Stree, T. S. Taylor, J. Tooker, M. Tupper, S. K. Wong, and S. Yamaguchi, in Proceedings of the 11th Conference on Plasma Physics and Controlled Fusion Research, 1986 (International Atomic Energy Agency, Vienna, 1987), Vol. I, p. 159.

${ }^{22}$ G. R. McKee, Bull. Am. Phys. Soc. 52, 350 (2007). 